\title{
A 34-year-old man with refractory heartburn and intermittent dysphagia to solids
}

\author{
Hoang Pham MSc, William H. Yang MD
}

\begin{abstract}
A 34-year-old man presents to his family physician after completing a two-month trial of a proton pump inhibitor for heartburn. The heartburn, which had begun three months earlier, has not responded to the treatment. The patient describes experiencing intermittent difficulty swallowing solids (e.g., bread and steak) and has tried to compensate by chewing food slowly and washing meals down with liquids to prevent food from sticking in his throat. He has not experienced weight loss and is not aware of food hypersensitivities. He has environmental allergies to tree pollens.
\end{abstract}

\section{What diagnoses should be considered?}

In the context of heartburn-dominant dyspepsia, dysphagia to solids is an alarm feature because it may indicate the presence of esophageal stenosis, strictures, rings, webs or malignant disease. ${ }^{1}$ However, eosinophilic esophagitis should also be considered in an atopic man less than 50 years of age who has a history of intermittent dysphagia to only solids with associated chronic heartburn. ${ }^{2}$ Eosinophilic esophagitis is a chronic immune/ antigen-mediated disease characterized by eosinophilic inflammation of the esophageal epithelium, resulting in esophageal dysfunction and fibrosis if left untreated. ${ }^{2}$ The pathogenesis is not fully understood yet but seems to be mixed in terms of both immunoglobulin E-mediated processes and delayed $\mathrm{Th} 2$ inflammation. ${ }^{2}$

\section{What questions should this patient be asked?}

History-taking plays an important role in characterizing dysphagia and narrowing the differential diagnosis, as per the Canadian Association of Gastroenterology dysphagia algorithm. ${ }^{3}$ The first step is to ask patients with dysphagia whether they have difficulty initiating a swallow (suggesting oropharyngeal dysphagia) or completing a swallow (suggesting esophageal dysphagia). Patients with oropharyngeal dysphagia may report a history of coughing, choking or nasal regurgita- tion, whereas those with esophageal dysphagia may complain of food sticking. ${ }^{3}$ The next step is to determine whether dysphagia is to solids, liquids or both. Dysphagia to only solids increases the likelihood of a mechanical lesion of the esophagus rather than a neuromuscular disorder. ${ }^{3}$

The onset and frequency of dysphagia, particularly whether it has been intermittent or progressive, provides further clues. Rapidly progressive dysphagia is more worrisome for a growing mass. ${ }^{3}$ Other worrisome features of possible malignant disease causing mechanical obstruction in the esophagus include age over 50 and unexplained weight loss. ${ }^{3}$

Several features on history-taking may further increase a clinical suspicion of eosinophilic esophagitis. According to a systematic review, patients with eosinophilic esophagitis usually have dysphagia to solids and elaborate coping mechanisms for the dysphagia. ${ }^{2}$ They may deliberately avoid highly textured foods such as meat, and bulky foods such as bagels. Other strategies include mincing food into small pieces, prelubricating foods with liquids or butter before eating, meticulous chewing with prolonged meal durations and washing food down with liquids. Any history of long-lasting dysphagia that resulted in food impaction requiring emergent intervention (e.g., endoscopic removal) substantially increases the likelihood of eosinophilic esophagitis. ${ }^{2}$

In addition to intermittent dysphagia, the patient in our case was also experiencing symptoms of gastrointestinal reflux disease (GERD) refractory to treatment with a proton pump inhibitor. Eosinophilic esophagitis is uncommon in refractory GERD in the absence of dysphagia or food impaction. Two prospective studies $(n=$ 68-105) showed that there was a low prevalence of eosinophilic esophagitis (0.9\%-8.8\%) among adults with symptoms of refractory GERD. ${ }^{4}$

Although about one-quarter of adults with eosinophilic esophagitis have symptoms consistent with GERD, dysphagia (93\%) and food
Competing interests: None declared.

This article has been peer reviewed.

The clinical scenario is fictional.

Correspondence to:

Hoang Pham,

hpham@uottawa.ca

CMAJ 2016. DOI:10.1503 /cmaj.150786 
impaction $(62 \%)$ are far more common. ${ }^{5}$ Two prospective studies involving about 300 participants reported that up to $15 \%$ of adults presenting with refractory GERD and dysphagia may have eosinophilic esophagitis, with the prevalence of the condition increasing to $48 \%$ among those with food impaction. ${ }^{4}$ A small prospective study involving 150 participants with refractory GERD reported that independent predictors of eosinophilic esophagitis in the six patients with diagnosed eosinophilic esophagitis were age less than 45 years, dysphagia and atopy. ${ }^{4}$

Given the patient's age, history of atopy, intermittent dysphagia and lack of response to treatment with a proton pump inhibitor, eosinophilic esophagitis is strongly suspected.

\section{Should this patient be referred for endoscopy?}

The patient should be referred for expedited endoscopy. The Canadian Association of Gastroenterology suggests that endoscopy be avoided for dyspepsia without alarm symptoms in patients less than 55 years of age (Box 1). ${ }^{6}$ However, dysphagia should be considered an alarm feature if difficulty swallowing solids is the primary symptom and it fails to respond to a two- to four-week trial of a proton pump inhibitor with once or twice daily standard dosing. ${ }^{1}$ Dysphagia unresponsive to even longer courses of proton pump inhibitors should prompt generalists to refer for an expedited endoscopy. ${ }^{1}$

Endoscopy is helpful for investigating a clinical history suspicious for mechanical obstruction, with the added benefit of being able to obtain biopsies. ${ }^{3}$

A diagnosis of eosinophilic esophagitis requires symptoms of esophageal dysfunction plus histologic evidence of predominantly eosinophilic inflammation ( $\geq 15$ eosinophils per high-power

Box 1: Choosing Wisely Canada
recommendation on endoscopy for
dyspepsia by the Canadian Association of
Gastroenterology
Avoid performing an endoscopy for dyspepsia
without alarm symptoms for patients under the
age of 55 years.
- Endoscopy is an accurate test for diagnosing
dyspepsia, but organic pathology that does
not respond to acid suppression or
Helicobacter pylori eradication therapy is
rare under the age of 55 . Most guidelines
therefore recommend as the first-line
approach for managing dyspepsia either
empirical proton pump inhibitor therapy or
a noninvasive test for $H$. pylori and then
offering therapy if the result is positive. If
the patient has alarm features such as
progressive dysphagia, anemia or weight
loss, endoscopy may be appropriate.

field) restricted to the esophagus that persist after a two-month trial of a proton pump inhibitor. ${ }^{7}$ Histology is required because no endoscopic features are considered pathognomonic. ${ }^{7}$ However, the authors of a clinical prediction tool for this condition prospectively enrolled 81 patients with eosinophilic esophagitis and 144 controls with GERD and dysphagia symptoms into a validation study. Certain clinical factors (age $<50 \mathrm{yr}$, male sex, presence of dysphagia and food allergies) combined with specific endoscopic features (presence of esophageal rings, furrows and plaques, and absence of hiatal hernia) predicted eosinophilic esophagitis with a sensitivity of $84 \%$, specificity of $97 \%$ and accuracy of $92 \% .{ }^{8}$ An online calculator developed by the University of North Carolina's Center for Esophageal Disease and Swallowing is available to help predict the probability of this condition (https://gicenter.med.unc. edu/cedas/eoe_clinical_calculator.html).

\section{Given the high likelihood of eosinophilic esophagitis, what information about its management should be discussed?}

Diet modification can be considered as an initial step in the management of eosinophilic esophagitis. ${ }^{7}$ A meta-analysis of 33 studies involving 1317 patients with the condition found that a six-food elimination diet (milk, soy, egg, nuts, seafood and wheat) was likely the best dietary approach for motivated adults. ${ }^{9}$ This approach balances the lower effectiveness of this diet (71.9\% in adults) against the major disadvantages of a more effective elemental diet (e.g., no table food, unpleasant taste, high cost, extremely limiting socially). ${ }^{9}$ There have been more liberal elimination diets developed, such as a four-food elimination diet (milk, wheat, egg and soy), a milk elimination diet and elimination diets directed by allergy testing, but they are less effective and less well studied. ${ }^{9}$ However, they may be considered for patients who desire a less restrictive diet or are having difficulty adhering to the six-food elimination diet.

Evidence from a recent systematic review and meta-analysis of seven randomized controlled trials involving 226 patients suggests that the use of swallowed inhaled steroids for an initial duration of eight weeks serves as first-line pharmacotherapy, after an initial trial of a proton pump inhibitor. ${ }^{10}$ Options include fluticasone $440-880 \mu \mathrm{g}$ twice daily or budesonide $1 \mathrm{mg}$ twice daily in adults. ${ }^{7}$ Fluticasone should be puffed directly into the mouth without inhaling or using a spacer and then dry swallowed. Budesonide can be swallowed as an oral viscous solution or nebulized mixture. Food and drink should be avoided for 30 minutes after administration of swallowed topical 
steroids. The most common adverse effect was an increased risk of asymptomatic esophageal candidiasis responsive to antifungal therapy.$^{10}$ Use of systemic steroids (e.g., prednisone $1 \mathrm{mg} / \mathrm{kg}$ daily) should be reserved for more severe cases. ${ }^{2,7}$

Based on large case series, endoscopic esophageal dilation may be considered in symptomatic adults with strictures refractory to diet and pharmacotherapy. ${ }^{?}$

An allergy consultation may be helpful in optimizing diet therapies and comorbid atopic conditions, which may contribute to the immunopathogenesis of this condition. ${ }^{2,7}$

Eosinophilic esophagitis is a chronic disease with frequent symptom recurrence after initial treatment. Patients should be counselled on the possible need for maintenance therapy (diet or pharmacotherapy) for controlling symptoms and preventing complications. Indications for maintenance therapy include narrow esophagus, prior stricture requiring repeated dilations, prior emergent endoscopy for food impaction, prior esophageal perforation, prior Boerhaave syndrome, severe or ongoing symptoms and patient preference. $^{7}$

A collaborative care model between the patient, consultant and primary care physician is important in the long-term management of eosinophilic esophagitis, because the condition requires continuing patient education, monitoring of adherence to and adverse effects of treatment, and monitoring for complications.

\section{Case revisited}

The patient was referred for upper endoscopy, and eosinophilic esophagitis was diagnosed after biopsies showed esophageal eosinophilia. Swallowed inhaled steroid therapy with fluticasone was prescribed. Because the patient wanted to follow the least restrictive diet modification strategy, allergy testing helped guide his dietary choices. The allergist also helped optimize his environmental allergies with nasal corticosteroids, antihistamines and subcutaneous immunotherapy. At one-year followup, the patient's symptoms had improved considerably, which motivated him to continue with his diet and fluticasone treatment to minimize mild ongoing symptoms.

\section{References}

1. Armstrong D, Marshall JK, Chiba N, et al. Canadian Consensus Conference on the management of gastroesophageal reflux disease in adults - update 2004. Can J Gastroenterol 2005;19:15-35.

2. Liacouras CA, Furuta GT, Hirano I, et al. Eosinophilic esophagitis: updated consensus recommendations for children and adults. J Allergy Clin Immunol 2011;128:3-20.e6.

3. Cockeram AW. Canadian Association of Gastroenterology practice guidelines: evaluation of dysphagia. Can J Gastroenterol 1998;12:409-13.

4. García-Compeán D, González-Cervera JA, Marrufo García CA, et al. Prevalence of eosinophilic esophagitis in patients with refractory gastroesophageal reflux disease symptoms: a prospective study. Dig Liver Dis 2011:43:204-8.

5. Sgouros SN, Bergele C, Mantides A. Eosinophilic esophagitis in adults: a systematic review. Eur J Gastroenterol Hepatol 2006; 18:211-7.

6. Canadian Association of Gastroenterology: Five things physicians and patients should question. Ottawa: Choosing Wisely Canada; 2014 Available: www.choosingwiselycanada.org/recommendations/ gastroenterology-2/ (accessed 2015 Dec. 4).

7. Dellon ES, Gonsalves N, Hirano I, et al. ACG clinical guideline: evidenced based approach to the diagnosis and management of esophageal eosinophilia and eosinophilic esophagitis (EoE). Am J Gastroenterol 2013;108:679-92.

8. Dellon ES, Rusin S, Gebhart JH, et al. A clinical prediction tool identifies cases of eosinophilic esophagitis without endoscopic biopsy: a prospective study. Am J Gastroenterol 2015;110:1347-54

9. Arias Á, González-Cervera J, Tenias JM, et al. Efficacy of dietary interventions for inducing histologic remission in patients with eosinophilic esophagitis: a systematic review and meta-analysis. Gastroenterology 2014;146:1639-48.

10. Chuang MY, Chinnaratha MA, Hancock DG, et al. Topical steroid therapy for the treatment of eosinophilic esophagitis (EoE): a systematic review and meta-analysis. Clin Transl Gastroenterol 2015;6:e82-9.

Affiliations: Faculty of Medicine (Pham, Yang), University of Ottawa; Ottawa Allergy Research Corporation (Yang), Ottawa, Ont.

Contributors: Hoang Pham drafted the manuscript, which both authors revised. Both authors approved the final version to be published and agreed to act as guarantors of the work.

Decisions is a series that focuses on practical evidence-based approaches to common presentations in primary care. The articles address key decisions that a clinician may encounter during initial assessment. The information presented can usually be covered in a typical primary care appointment. Articles should be no longer than 650 words, may include one box, figure or table and should begin with a very brief description (75 words or less) of the clinical situation. The decisions addressed should be presented in the form of questions. A box providing helpful resources for the patient or physician is encouraged. 